\title{
IGF-1R tyrosine kinase inhibitors and Vitamin K1 enhance the antitumor effects of Regorafenib in HCC cell lines
}

\author{
Maria Grazia Refolo ${ }^{1, *}$, Rosalba D'Alessandro ${ }^{1, *}$, Catia Lippolis ${ }^{1}$, Nicola Carella ${ }^{1}$, \\ Aldo Cavallini ${ }^{1}$, Caterina Messa ${ }^{1}$ and Brian Irving Carr ${ }^{2}$ \\ 1'Laboratory of Cellular and Molecular Biology, Department of Clinical Pathology, National Institute of Gastroenterology, "S. \\ De Bellis" Research Hospital, Castellana Grotte, BA, Italy \\ ${ }^{2}$ Visiting Professor, Program for Targeted Experimental Therapeutics, Izmir Biomedicine and Genome Center, Dokuz Eylul \\ University, Izmir, Turkey \\ *These authors have contributed equally to this work \\ Correspondence to: Brian Irving Carr, email: brianicarr@hotmail.com \\ Caterina Messa, email: caterina.messa@irccsdebellis.it
}

Keywords: combination therapy, synergism, regorafenib, vitamin K1, insulin-like growth factor receptor

Received: June 16, $2017 \quad$ Accepted: September 13,2017 Published: September 30, 2017

Copyright: Refolo et al. This is an open-access article distributed under the terms of the Creative Commons Attribution License 3.0 (CC BY 3.0), which permits unrestricted use, distribution, and reproduction in any medium, provided the original author and source are credited.

\section{ABSTRACT}

The recent RESORCE trial showed that treatment with Regorafenib after Sorafenib failure provided a significant improvement in overall survival in HCC patients. Preclinical and clinical trial data showed that Regorafenib is a more potent drug than Sorafenib. In this study we aimed at improving Regorafenib actions and at reducing its toxicity, by targeting parallel pathways or by combination with Vitamins K (VKs). We investigated the effects of Regorafenib administrated at low concentrations and in combination with either VK1 and/or with GSK1838705A or OSI-906, two IGF1-R inhibitors, on HCC cell growth and motility. Our results showed that both IGF1-R inhibitors potentiated the antiproliferative and pro-apoptotic effects of Regorafenib and/or VK1 in HCC cell lines. Moreover we provide evidence that the combined treatment with IG1-R antagonists and Regorafenib (and/or VK1) also caused a significant reduction and depolymerization of actin resulting in synergistic inhibition exerted on cell migration. Thus, simultaneous blocking of MAPK and PI3K/ Akt cascades with IGF1-R inhibitors plus Regorafenib could represent a more potent approach for HCC treatment.

\section{INTRODUCTION}

Hepatocellular carcinoma (HCC) is the second most frequent cause of cancer-related death worldwide [1]. The multikinase inhibitor Sorafenib is the only systemic drug approved for the first-line treatment of advanced HCC. Although Sorafenib treatment provides a significant improvement in overall survival, a high percentage of Sorafenib-treated patients experience disease progression and major toxicity [2].

Recently, the RESORCE trial showed that treatment with Regorafenib (Fluoro-Sorafenib) provided a significant improvement in overall survival in patients with $\mathrm{HCC}$ who had disease progression during first-line treatment with
Sorafenib. Median overall survival was 10.6 months in the Regorafenib group compared with 7.8 months in those receiving placebo, representing a significant reduction in the risk for death [3]. The significance of this is that Regorafenib exerted responses and survival benefits even in Sorafenib resistant patients. Thus, these two drugs must be non-identical in their actions.

Regorafenib is also an oral multikinase inhibitor, that has action against cRAF/RAF-1, B-RAF, PDGF and c-Kit [4-6]. However, despite the availability of these targeted kinase inhibitors, drug toxicity and resistance and subsequent disease relapse remain a problem in HCC management. Several studies have aimed at improving the actions of these systemic drugs and at reducing their 
toxicity, such as by targeting parallel pathways [7] or by combination with Vitamins K (VKs) [8-9]. VKs are fatsoluble vitamins with minimal toxicity, that play a major role not only in blood coagulation and bone metabolism [10], but also have anti-tumor activity in different experimental tumor types [11-13]. Many mitogens have been shown to be important for HCC growth. Amongst them there is IGF1 [14] and we have shown that inhibition of IGF1-R antagonizes HCC cell lines growth [15]. In the current work we extend these previous observations to show that addition of IGF1-R inhibition enhances the effects of Regorafenib as well as its combination with $\mathrm{VK}$, and in addition permits the use of lower Regorafenib concentrations, that might result in lower toxicity.

\section{RESULTS}

\section{GSK1838705A and OSI-906 potentiate the antiproliferative effects of Regorafenib and/or VK1 in HCC cell lines}

We initially examined whether addition of IGF1-R inhibition could enhance Regorafenib effect. A range of concentrations was examined for each of the drugs that were studied (data not shown) and the lowest concentration of each drug that had a biological effect was subsequently used in all assays. PLC/PRF/5, HepG2 or HLF cells were cultured in presence of Regorafenib and/ or VK1. Cells that were treated with Regorafenib and/or VK1 were also cultured with or without GSK1838705A or OSI-906, two different IGF1-R inhibitors. Cell proliferation was evaluated after $48 \mathrm{~h}$ by MTT assay. Addition of VK1 exerted a potent inhibitory effect on cell growth in all the combinations of drugs analyzed. In particular, we found that VK1 enhanced the inhibitory effect of Regorafenib. Furthermore, the addition of this natural compound permitted the growth inhibition by Regorafenib at a concentration that was ten times lower than its IC50. In addition, this inhibitory effect was further potentiated by the combination with either GSK1838705A or OSI-906 (Figure 1). A combination index (CI) was computed for these combinations. Average CI values computed between the different cell lines were 0.5 and 0.7 for GSK1838705A/Regorafenib/VK1 and OSI-906/ Regorafenib/VK1 combinations respectively. These values were found to be well below the line of additivity $(\mathrm{CI} \leq 1)$, showing that synergy was involved in these drug interactions (data not shown).

\section{AFP response}

Since AFP is an important clinical tumor marker for HCC growth and aggressiveness, we examined the effects of GSK1838705A and OSI-906 on Regorafenib/VK1mediated inhibition of AFP secretion levels after $48 \mathrm{~h}$, using the two AFP-secreting human $\mathrm{HCC}$ cell lines, PLC/
$\mathrm{PRF} / 5$ and HepG2. Despite the significant reduction in the medium AFP levels in cells treated with the combination of Regorafenib/VK1, the enhancement exerted by further addition of IGF1-R inhibitors was weak (Figure 2A).

These results were confirmed also by Western blot analysis for cellular AFP expression (Figure 2B).

\section{GSK1838705A and OSI-906 potentiate the pro- apoptotic effects of Regorafenib and/or VK1 in HCC cell lines}

We next investigated the effects of GSK1838705A or OSI-906 on Regorafenib/VK1-mediated apoptosis after $48 \mathrm{~h}$, using the same experimental conditions as for the growth experiments. VK1 added simultaneously to Regorafenib $(0.1 \mu \mathrm{M}$ for HepG2 and $1 \mu \mathrm{M}$ for both PLC/ $\mathrm{PRF} / 5$ and HLF) caused an average increase of $44 \%$ in cellular Annexin V compared with Regorafenib treated cells. The percentage of apoptotic cells was further increased of $74 \%$ with GSK 1838705 A and of $100 \%$ with OSI-906 compared with the combination Regorafenib and VK1 (Figure 3A). These findings were confirmed by the evaluation of apoptotic status based on Caspase-3 and -7 activation analyzed by the Muse Caspase-3/7 assay (Figure 3B).

\section{GSK1838705A and OSI-906 potentiate the inhibitory effects of Regorafenib and/or VK1 in HCC cell migration and on actin polymerization}

To test the effects of GSK1838705A and OSI906 on Regorafenib/VK1-mediated inhibition of cell migration, cells were seeded onto Oris plates and treated with drugs according to the experimental conditions above. Cell migration assay was performed on wells coated with Collagen I and Fibronectin matrix and microscopic analysis was assessed both immediately after the stoppers removal (T0) and different later times, in the graphs were reported the percentage of migration after $48 \mathrm{~h}$. We found that the inhibition exerted by Regorafenib on migration was strongly potentiated with either VK1 and IGF1-R inhibitors and that these effects were independent of the matrix used. Interestingly, all the combination treatments showed synergistic effects (CI $<1$, data not shown) in driving the inhibition of cell migration compared to the single agent (Figure 4A). We next examined whether treatment of cells with these drug combinations affected the actin cytoskeleton. Cells that were stained with DyLight 554 Phalloidin revealed that both Regorafenib and VK1, administrated for $24 \mathrm{~h}$ alone or in combination with GSK1838705A or OSI-906, caused significant reduction and depolymerization of actin in the cells on Fibronectin matrix. This resulted in a loss of F-actin fibers in the cytoplasm and their redistribution around the nucleus, whereas in control cells actin fibers were diffusely distributed in the cytoplasm (Figure 4B). 


\section{GSK1838705A and OSI-906 potentiate the} inhibition by Regorafenib of MAPK and PI3K/ Akt signaling

We have previously shown that Regorafenib and GSK1838705A decrease the levels of p-IGF1-R (15) and therefore now wished to examine the downstream signaling. Cells treated with Regorafenib and/or VK1 were additionally cultured with or without GSK1838705A or OSI-906. MAPK phosphorylation was evaluated relative to the total MAPK expression by Muse MAPK Activation Dual Detection Kit. Addition of VK1 to the cultures caused an additional decrease of $18 \%$ in pERK levels, compared with Regorafenib alone (12\%) used at low concentrations (Figure 5A). Both IGF1-R antagonists further enhanced this inhibitory effect when either was added in combination with Regorafenib and VK1, and caused a decrease of $62 \%$ in pERK levels, using GSK $1838705 \mathrm{~A}$ and a decrease of $60 \%$, using OSI906. Using Muse PI3K Activation dual detection Kit, we investigated the activation of PI3K/Akt pathway in the same experimental conditions. We found that neither Regorafenib nor VK1 had any effect on the percentage of p-Akt (Ser473) activation, but when these two drugs

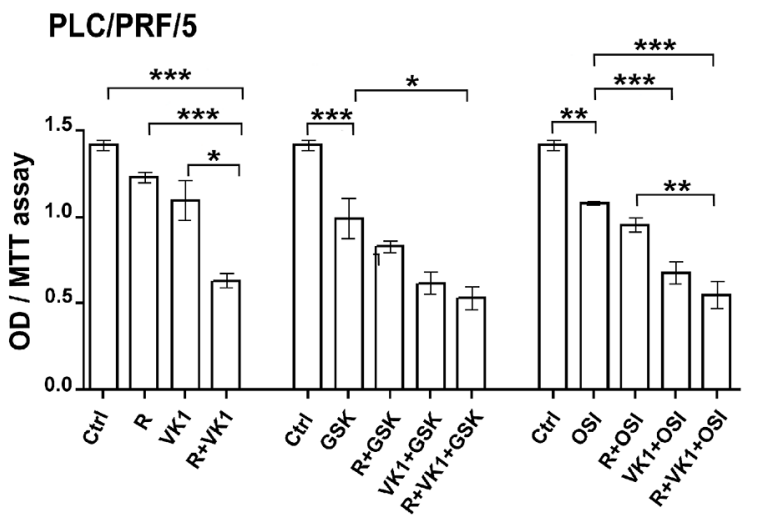

\section{HLF}

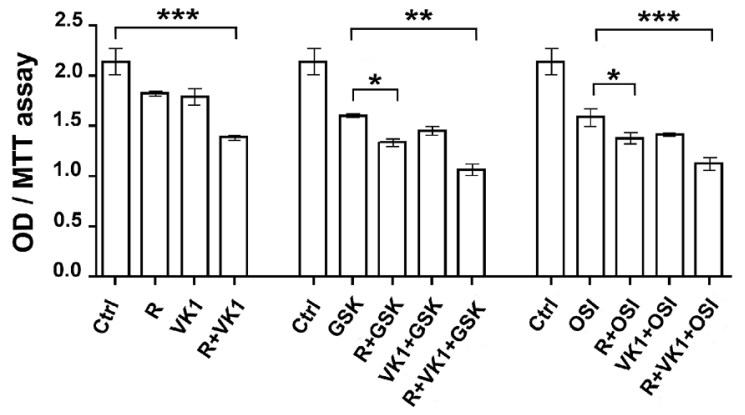

\section{HepG2}

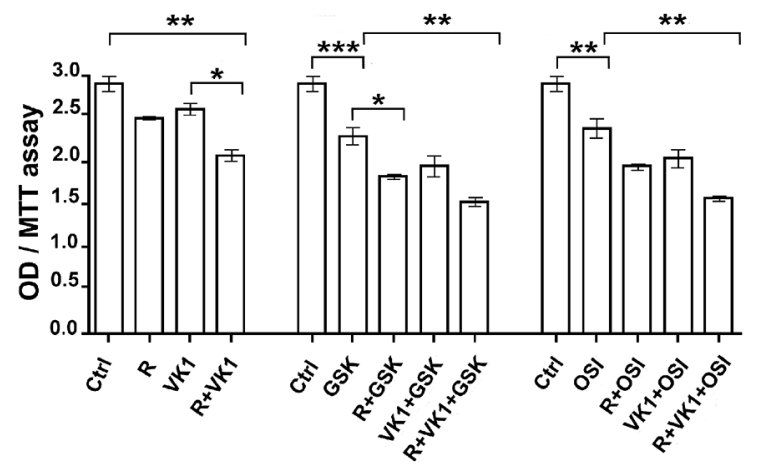

Figure 1: GSK1838705A and OSI-906 potentiate the antiproliferative effects of Regorafenib and/or VK1 in HCC cells. Cells were cultured with $1 \mu \mathrm{M}(\mathrm{PLC} / \mathrm{PRF} / 5)$ or $0.1 \mu \mathrm{M}$ (HepG2) Regorafenib, $25 \mu \mathrm{M}$ VK1, $4 \mu \mathrm{M}$ GSK1838705A and $0.5 \mu \mathrm{M}$ OSI906 administrated singularly or in combination. MTT assay was performed after $48 \mathrm{~h}$. The results of three independent experiments are expressed as means $\pm \mathrm{SD} .{ }^{*} \mathrm{p}<0.05 ;{ }^{* *} \mathrm{p}<0.001 ;{ }^{* * *} \mathrm{p}<0.0001$. 
were added in combination, we found a decrease of $15 \%$ in p-Akt levels with respect to untreated cells. An even greater reduction in $\mathrm{p}$-Akt was obtained by further addition of either of the IGF1-R inhibitors. GSK1838705A and OSI-906, which caused a further decrease of $10 \%$ and $50 \%$ respectively in p-Akt levels when they were added in combination with Regorafenib and VK1 (Figure 5B). Western Blot analysis of cell lysates in the same experimental conditions was performed to measure the phosphorylation levels of several proteins involved in

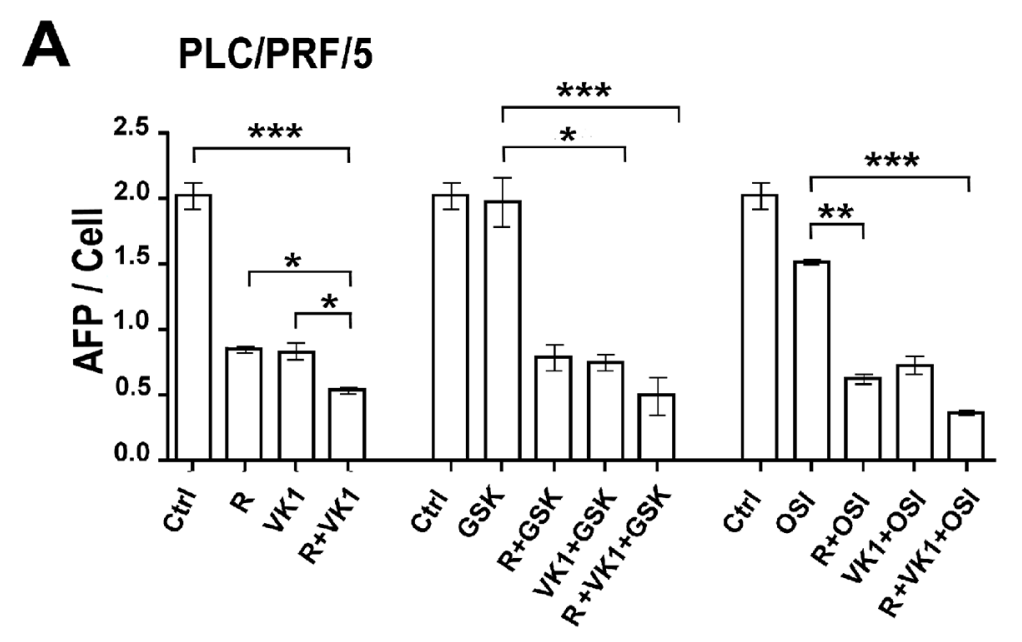

HepG2

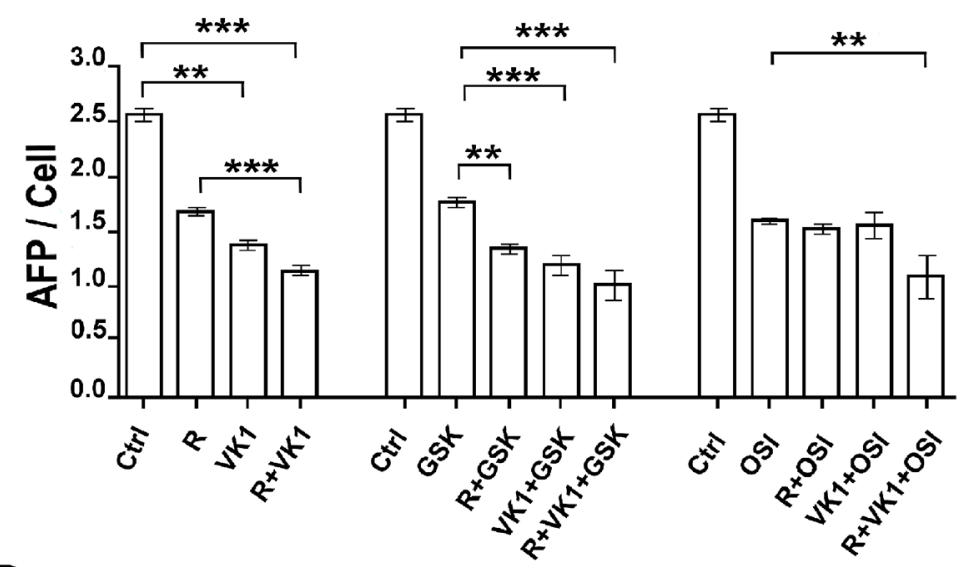

B
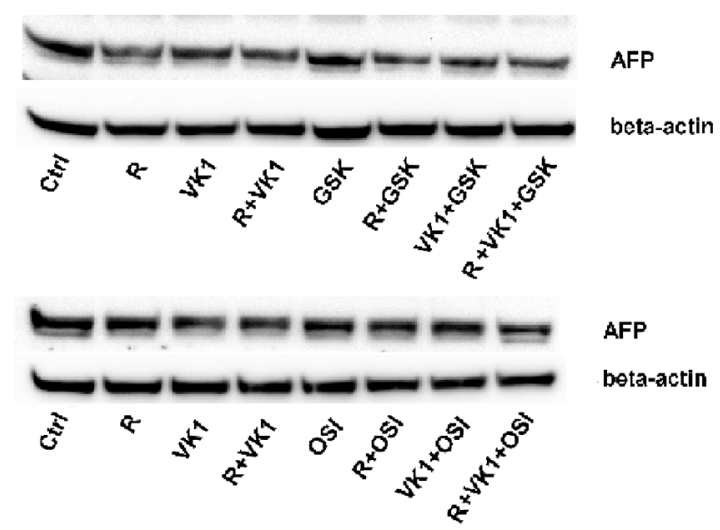

Figure 2: GSK1838705A and OSI-906 potentiate the Regorafenib/VK1-mediated inhibition of AFP secretion. (A) AFP levels in the cell culture medium of PLC/PRF/5 and HepG2 cell lines and the number of viable cells after treatment of $48 \mathrm{~h}$ with $1 \mu \mathrm{M}(\mathrm{PLC} / \mathrm{PRF} / 5)$ or $0.1 \mu \mathrm{M}$ (HepG2) Regorafenib, 25 $\mu \mathrm{M}$ VK1, $4 \mu \mathrm{M}$ GSK1838705A and $0.5 \mu \mathrm{M}$ OSI-906 administrated singularly or in combination. (B) Western blotting analysis of cellular AFP in PLC/PRF/5 cells. The experiments were repeated three times. The results of three independent experiments are expressed as means \pm SD. ${ }^{*} \mathrm{p}<0.05 ;{ }^{* *} \mathrm{p}<0.001 ;{ }^{* * *} \mathrm{p}<0.0001$. 
MAPK and PI3K signaling. The expression levels of p-p38 and p-JNK, which are involved in MAPK signaling, were decreased in cells treated both with Regorafenib/VK1 and with Regorafenib/VK1/IGF1-R inhibitor combinations. By contrast, the expression levels of p-TSC2 and p-S6, which are involved in PI3K/Akt signaling, were significantly reduced only in Regorafenib/VK1 treated cells when they were also treated with IGF1-R inhibitors (Figure 5C-5D).

\section{DISCUSSION}

The outcome of the recent RESORCE trial was that Regorafenib treatment provided survival benefit in patients with $\mathrm{HCC}$, after progression on Sorafenib treatment [3]. These results suggest that the sequential use of molecules with tyrosine-kinase inhibitor activity can provide a significant improvement in overall survival. Preclinical and trial data highlighted that Regorafenib with respect to its analog Sorafenib, is a more potent drug, that is may be able to inhibit a distinct set of kinases, including the angiogenic and stromal receptors VEGFR-1-3, TIE-2, FGFR1 and PDGF- $\beta$ as well as the oncogenic receptors KIT and RET [5, 16-18]. However, drug toxicity and the presence of resistance mechanisms in the majority of patients [19] remain a problem in HCC treatment. Our previous in vitro studies suggested that microenvironmental factors can promote resistance to multikinase inhibitors drugs or chemotherapy $[15,20]$.
A
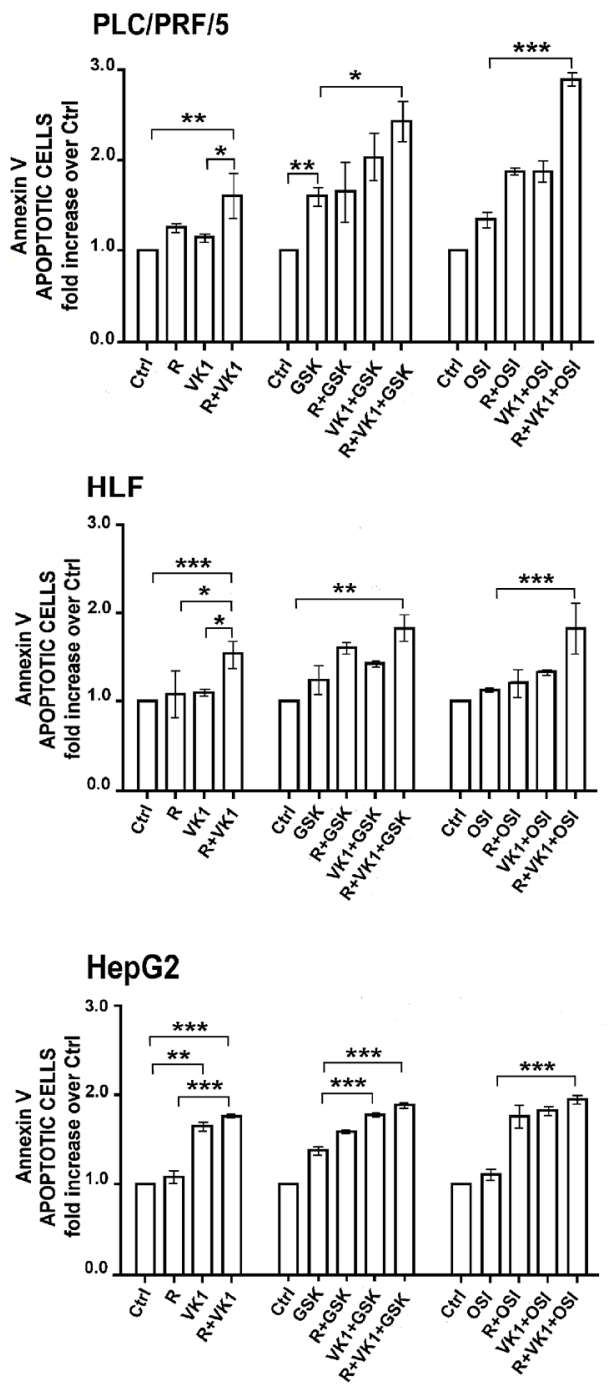

B
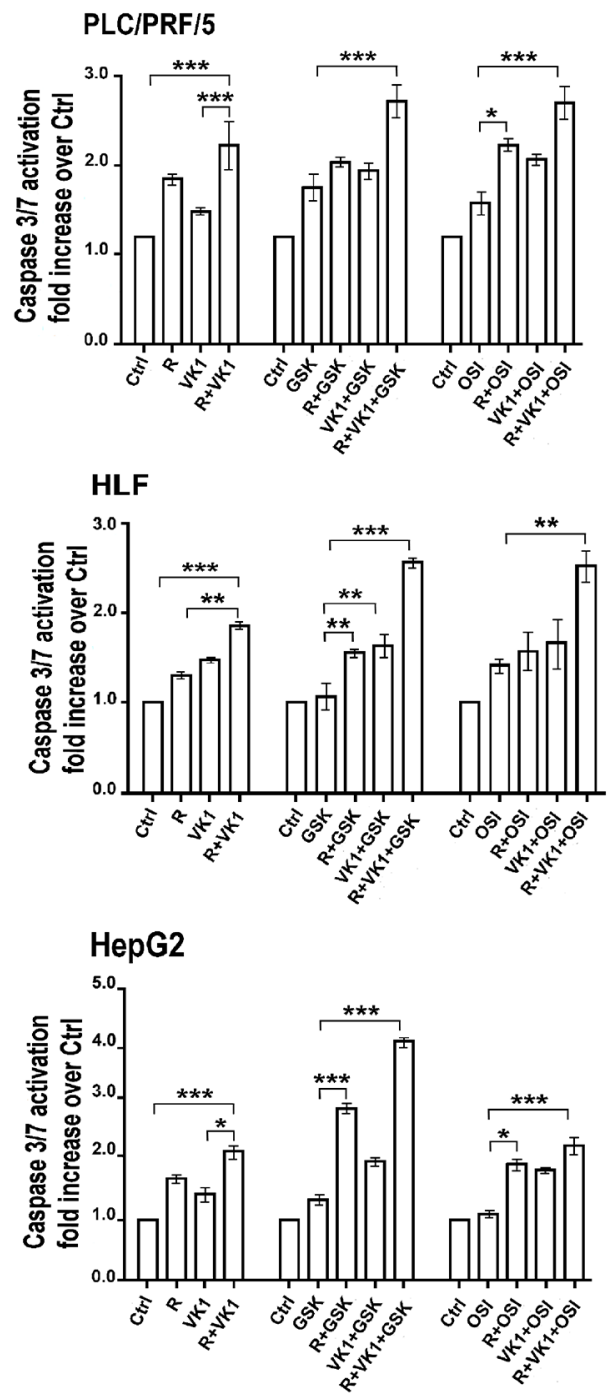

Figure 3: GSK1838705A and OSI-906 potentiate the pro-apoptotic effects of Regorafenib and/or VK1 in HCC cell lines. Cells were cultured with $1 \mu \mathrm{M}$ (PLC/PRF/5) or $0.1 \mu \mathrm{M}$ (HepG2) Regorafenib, $25 \mu \mathrm{M}$ VK1, $4 \mu \mathrm{M}$ GSK1838705A and $0.5 \mu \mathrm{M}$ OSI-906 administrated singularly or in combination. Muse Annexin V (A) and Muse Caspase-3/7 (B) Cell Assays were performed after 48 h. The results of three independent experiments are expressed as means \pm SD. ${ }^{*} \mathrm{p}<0.05 ;{ }^{* *} \mathrm{p}<0.001 ;{ }^{* * *} \mathrm{p}<0.0001$. 
The studies of the resistance mechanisms to Sorafenib have revealed that in tumors with acquired resistance, there is an over expression of IGF1 and its major downstream pathway PI3K-Akt [21]. Moreover, we previously reported that platelet-associated IGF1 antagonized Regorafenib-mediated growth, migration and invasion inhibition, as well as the drug-mediated induction of apoptosis in HCC cells in vitro [15], suggesting common

A
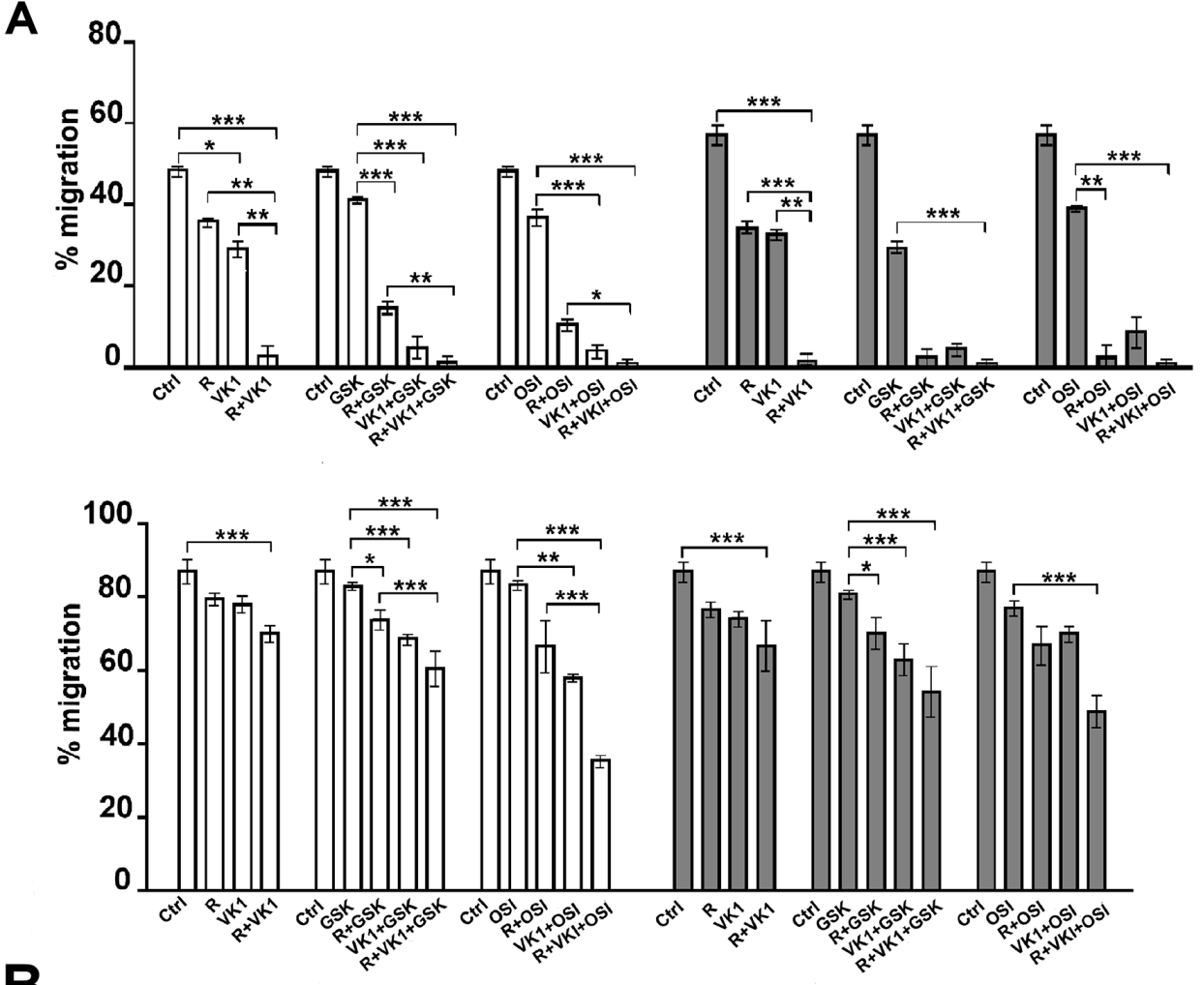

B
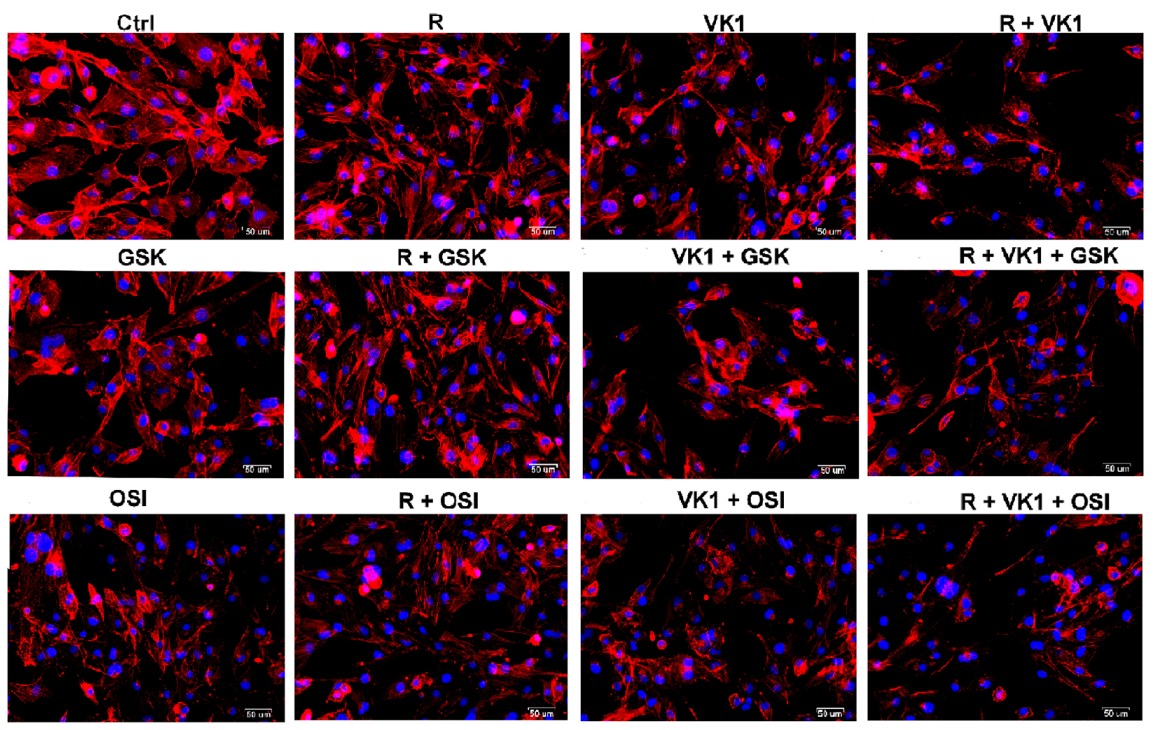

Figure 4: GSK1838705A and OSI-906 enhance the inhibitory effects of Regorafenib and/or VK1 on depolymerization of actin cytoskeleton and cell migration. (A) Migration assay was performed in PLC/PRF/5 and HLF cells seeded on collagen I and fibronectin coated wells and treated as described. The microscopic analysis was assessed at the time T0 and T2 (after $48 \mathrm{~h}$ ). The values were expressed as percentage of migration, where $100 \%$ represents the detection zone completely closed. The results of three independent experiments, expressed as mean $\pm \mathrm{SD}$, are plotted in the relative graph. ${ }^{*} \mathrm{p}<0.05 ;{ }^{* *} \mathrm{p}<0.001 ;{ }^{* * *} \mathrm{p}<0.0001$. (B) HLF cells stained with DyLight 554 Phalloidin after treatment with $1 \mu \mathrm{M}$ Regorafenib, $25 \mu \mathrm{M}$ VK1, $4 \mu \mathrm{M}$ GSK1838705A and $0.5 \mu \mathrm{M}$ OSI-906 administrated singularly or in combination. Scale bar: $50 \mu \mathrm{m}$. 
escape mechanisms for both Sorafenib and Regorafenib. Hence, simultaneous blocking of MAPK and PI3K/ Akt cascades with IGF1-R inhibitors and Regorafenib could represent a crucial approach for HCC treatment. The strategies to block IGF1-R include three different classes of drugs: monoclonal anti-IGF1-R antibodies, small molecules tyrosine kinase inhibitors (TKIs) and IGF ligand antibodies. Currently, several anti-IGF1-R antibodies and TKI molecules have demonstrated that these targeting approaches can induce strong anti-tumor activities and are under clinical investigation. Among TKIs, OSI-906 and BMS754807 are the most specific and act on the intra-cellular domain of IGF1-R. Others, such as GSK1838705A, act on the extra-cellular domain [22]. Moreover, we found that GSK1838705A strongly

A

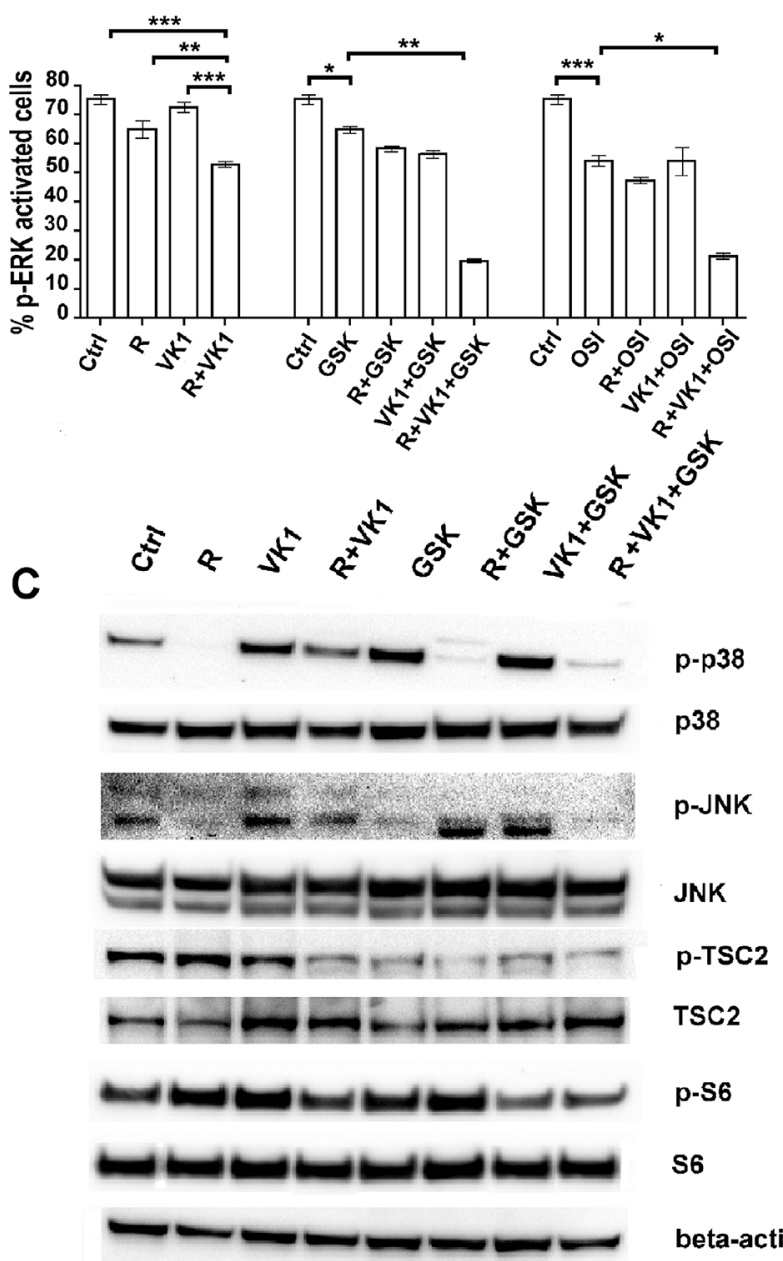

enhanced Regorafenib action [15], showing that the IGF1-R and PI3K/Akt/mTOR signaling pathways are associated with HCC biology [15, 23, 24]. Since both Sorafenib and Regorafenib have multiple toxicities and a large percent of patients need to be dose-reduced or stop taking the drugs, our previous studies had underlined that the combined administration of both VK1 and Sorafenib drastically reduced the drug dosage that was required for growth inhibition in several HCC cell lines [9, 11, 33]. Moreover, non-toxic VK1 could inhibit any recovery of growth and migration in Regorafenib pre-treated HCC cells [25]. Overall this knowledge led us to investigate the effects of Regorafenib administrated at low concentrations and in combination with either VK1 and the IGF1-R inhibitors on HCC cells growth and motility. In the present

B
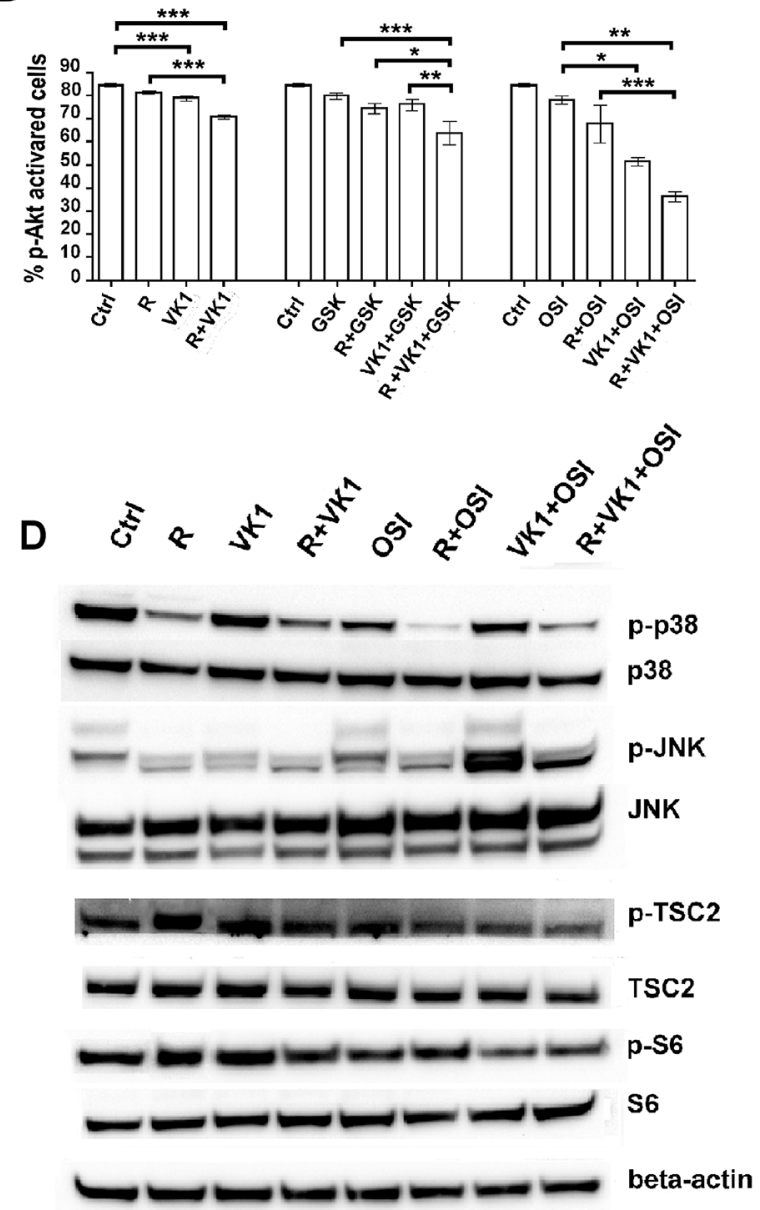

Figure 5: Effects of the combination Regorafenib/VK1 and IGF1-R inhibitors on the modulation of MAPK and

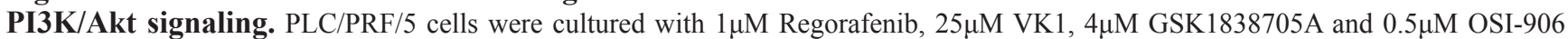
administrated singularly or in combination. (A) The Muse MAPK Activation Kit was used to evaluate the MAPK phosphorylation relative to the total MAPK expression after $15 \mathrm{~min}$. The results of three independent experiments, expressed as mean $\pm \mathrm{SD}$, are plotted in the relative graph. (B) The Muse PI3K Activation dual detection Kit was used to detect the Akt phosphorylation (Ser473) relative to the total Akt expression after $24 \mathrm{~h}$. The results of three independent experiments, expressed as mean $\pm \mathrm{SD}$, are plotted in the relative graph. ${ }^{*} \mathrm{p}<0.05$; ${ }^{* *} \mathrm{p}$ $<0.001 ;{ }^{* * *} \mathrm{p}<0.0001$. (C-D) Western Blot showing the expression levels of some proteins involved in MAPK and PI3K/Akt pathways after $48 \mathrm{~h}$ of single or combined treatments. 
study we found that VK1 enhanced the inhibitory effect of Regorafenib on cell proliferation. Furthermore, the addition of this natural compound allowed the use of a concentration of Regorafenib that was ten times lower than its IC50. The inhibitory effect was further potentiated by the combination with either of the IGF1-R inhibitors used, GSK1838705A and OSI-906. The combination indexes $(\mathrm{CI})$ computed for these drug combinations were found to be well below the line of additivity ( $\mathrm{CI} \leq 1)$, showing that drug synergy was likely involved in these drug interactions. Since AFP is an important clinical tumor marker for HCC growth and aggressiveness, we examined the effects of GSK1838705A and OSI-906 on Regorafenib/VK1-mediated inhibition of AFP secretion levels in HCC cell lines. These experiments revealed a significant reduction in the medium AFP levels in cells treated with the combination of Regorafenib and VK1, whereas the enhancement exerted by further addition of IGF1-R inhibitors was weak, suggesting a major involvement of MAPK signaling. Synergistic effects obtained by combining GSK1838705A or OSI-906 with Regorafenib/VK1 were also clearly visible in the induction of apoptosis. VK1 added simultaneously to cells with Regorafenib caused an average increase of $44 \%$ compared with Regorafenib treated cells, and this percentage was further enhanced by the addition of GSK1838705A (74\%) or OSI-906 (100\%).

Previous studies have shown that IGF1 had a protective effect on Sorafenib- or VK1-mediated reduction and depolymerization of actin cytoskeleton, with ensuing effects on cell motility [15, 23, 24]. Here, we provide evidence that the combined treatment with IGF1-R antagonists and Regorafenib and/or VK1 caused a significant reduction and depolymerization of actin, resulting in synergistic inhibition of cell migration. These data are consistent with those of Western Blot analysis, in which we evaluated the phosphorylation level of some of the principal proteins involved in the MAPK and $\mathrm{PI} 3 \mathrm{~K} / \mathrm{Akt}$ signaling cascades which are involved in cell proliferation, oncogenesis, differentiation, inflammation and stress responses [26].

These experiments are the first to show the enhancement of the growth-inhibitory actions of Regorafenib by the totally non-toxic (to humans) vitamin $\mathrm{K}$ [34], as well as by IGF1-R inhibitors. Given the considerable toxicity in patients of both Sorafenib and Regorafenib, combinations with other agents that can enhance its effectiveness and thus permit lower clinical doses to be used, are of potential clinical interest.

Overall these results indicate that therapies based on the combination of agents with additive or synergistic activity could represent new therapeutic strategies against $\mathrm{HCC}$ and might permit the use of lower and less toxic Regorafenib doses.

\section{MATERIALS AND METHODS}

\section{Cells and drugs}

PLC/PRF/5, HepG2 and HLF human HCC cell lines were purchased from the National Institute of Biomedical Innovation JCRB Cell Bank (Osaka, Japan). The culture medium was Dulbecco's Modified Eagle's Medium (DMEM). All cell culture components were purchased from Sigma-Aldrich (Milan, Italy). Regorafenib was gifted from Bayer Corp (West Haven, CT USA ); vitamin K1 was purchased from International Medication Systems, Limited (So. El Monte, CA, USA), GSK1838705A and S1091 Linsitinib (OSI-906) from Selleckchem (Houston, TX, USA).

\section{Cell culture}

PLC/PRF/5, HepG2 and HLF cell lines were cultured in DMEM in monolayer culture, and supplemented with 10\% fetal bovine serum (FBS), 100U/ $\mathrm{ml}$ penicillin, $100 \mu \mathrm{g} / \mathrm{ml}$ streptomycin, and incubated at $37^{\circ} \mathrm{C}$ in a humidified atmosphere containing $5 \% \mathrm{CO}_{2}$ in air. Since PLC/PRF/5 cells express high affinity IGF1-R that may mediate the stimulatory effects of exogenous IGF1 [27], we chose to investigate the MAPK activation and PI3K/Akt signaling markers only in this cell line.

\section{Cell proliferation and drug synergy evaluation}

The cells were cultured in medium containing different concentrations of Regorafenib $(1,2.5,5 \mu \mathrm{M})$ and/or VK1 $(6,12,25 \mu \mathrm{M})$ in presence of GSK1838705A $(2,4,6 \mu \mathrm{M})$ or OSI-906 $(0.13,0.5,1 \mu \mathrm{M})$. After $48 \mathrm{~h}$ of incubation, the proliferative response was estimated by colorimetric 3-(4,5 di-methylthiazol-2-yl)-2,5diphenyltetrazolium bromide (MTT) test. The trypan blue exclusion test was used to evaluate cell viability. Each experiment was performed in triplicate and repeated three times.

The potential synergistic, additive or antagonistic effects of Regorafenib or VK1 plus GSK1838705A or OSI906 used in combination were assessed experimentally and computationally using methods as described by Chou and Chou and Talalay [28, 29], implemented in CompuSyn software (Biosoft, UK). The Chou and Talalay approach takes into account drug potency as well as the relationship between dose and response for each drug. Results are reported as the Combination Index (CI). Values of $\mathrm{CI}<1, \mathrm{CI} \pm 1$, and $\mathrm{CI}>1$ imply synergy, additivity, and antagonism, respectively. An isobologram, based on an extension of the Lowe additivity model is also provided [30]. Similar to the CI values, values on the isobologram below the line of additivity represent combinations where 
synergy is present, values close to the line would represent additivity, and values above the line represent antagonistic effects. Moreover the Dose Reduction Index (DRI) was computed using CompuSyn software, this value denotes how many fold of dose reduction is allowed for each drug due to synergism when compared with the dose of each drug alone. In all the subsequent combined treatments each drug was used at the concentration showing above $\mathrm{CI}<1$, in particular cells were cultured with $1 \mu \mathrm{M}$ (PLC/ $\mathrm{PRF} / 5$ ) or $0.1 \mu \mathrm{M}$ (HepG2) Regorafenib, $25 \mu \mathrm{M}$ VK1, $4 \mu \mathrm{M}$ GSK1838705A and 0.5 $\mu \mathrm{M}$ OSI-906 administrated singularly or in combination.

\section{AFP measurement}

The cells were cultured as described in the proliferation paragraph. Medium AFP levels were measured by use of an automated system (UniCel Integrated WorkstationsDxC 660i, Beckman-Coulter, Fullerton, CA) using a chemiluminescent immunometric method. Sample measurements over the calibration range were automatically re-analyzed according to constructor's instructions.

\section{Apoptosis assay}

The cells were cultured as described in the proliferation paragraph. Two different apoptosis experiments were performed. Flow cytometry technology (Muse Cell Analyzer, Millipore, Darmstadt, Germany) was used to detect the fluorescent signal emitted by dye conjugated antibodies. The Muse Annexin V/Dead Cell Assay Kit (Millipore, Darmstadt, Germany) for quantitative analysis of live, early/late apoptotic and dead cells was used with a Muse Cell Analyzer. Briefly, the assay utilizes Annexin V to detect PS on the external membrane of apoptotic cells. A dead cell marker (7-AAD) is also used. The cells were then processed as described in the user's guide. Moreover, the simultaneous evaluation of apoptotic status based on Caspase- 3 and -7 activation and cellular plasma membrane permeabilization (cell death) was analyzed by the Muse Caspase-3/7 kit (Millipore). The assay provides relative percentage of cells that are live, early/late apoptotic or dead. Cells were processed according to the user's guide.

\section{Migration assay}

The cells were cultured as described in the proliferation paragraph. The migration assay was performed by Oris Cell Migration Assay (Platypus Technologies, Madison USA) that provided wells coated with Collagen I, Fibronectin or tissue culture treated. This TriCoated sytem permits the evaluation of the best surface coating for HCC cell lines used. Briefly, the cells seeded onto Oris plates have been to adhere on their surface except in the circle covered with a stopper (detection zone) and were subjected to different drug treatments. When the stoppers have been removed the cells started to migrate into the detection zone. Cells were examined microscopically throughout the incubation period to monitor progression of migration in the detection zone. Migration time was depended upon cell type, type of coating and specific drug treatment. Photographs were taken of each well immediately after the stoppers removal (T0) and after $24 \mathrm{~h}$ (T1), $48 \mathrm{~h}$ (T2) and $72 \mathrm{~h}$ (T3).

The values were expressed as percentage of migration, with $100 \%$ being when the detection zone was completely closed. The results were representative of three independent experiments. The relative graphs were created with GraphPad Prism 5.0 software.

\section{Immunofluorescence}

PLC/PRF/5 and HLF cells were cultured for $24 \mathrm{~h}$ in $1 \% \mathrm{FBS}$ medium and then were trypsinized, resuspended in $1 \%$ FBS medium and seeded in 96 multi-well plates. Experiments were performed as described previously [31]. Specifically, cell monolayers in 96 multi-well plates were fixed with Cytofix fixation buffer (BD biosciences, Milan, Italy) 10 minutes at room temperature and quenched in $0.1 \mathrm{M}$ glycine, then permeabilized for $20 \mathrm{~min}$ in PBS containing $0.1 \%$ Triton $\mathrm{X}-100$, blocked for $30 \mathrm{~min}$ at room temperature in BlockAid solution (Life technologies, Eugene, OR, USA) and immunolabeled in humidified dark chamber for $2 \mathrm{~h}$ with DyLight 554 Phalloidin (Cell Signaling, Beverly, MA, USA). Nuclei were stained with DAPI for $10 \mathrm{~min}$ in the dark. After rinsing with PBS, images were acquired with ZOE fluorescent cell imager (Bio-Rad, Milan, Italy).

\section{MAPK activation assay}

Muse MAPK Activation Dual Detection Kit (Millipore, Darmstadt, Germany), including a phosphospecific antiphospho-Erk1/2 (Thr202/Tyr204, Thr185/ Tyr187)-Phycoerythrin and an anti-Erk1/2-PECy5 conjugated antibody, was used to measure total levels of Erk. This two-color kit is designed to measure the extent of MAPK phosphorylation relative to the total MAPK expression in the cell population processed as described in the user's guide. The levels of both the total and phosphorylated protein can be measured simultaneously in the same cell, resulting in a normalized measurement of MAPK activation after stimulation. PLC/PRF/5 cell line was cultured in presence of the indicated concentrations of Regorafenib, VK1, GSK1838705A and OSI-906 alone or in combination for $15 \mathrm{~min}$. Results were representative of three independent experiments. The relative graphs were created with GraphPad Prism 5.0 software. 


\section{PI3K activation assay}

Muse PI3K Activation dual detection Kit was used to detect the fluorescent signal emitted by dye conjugated antibodies, a specific anti-phospho-Akt (Ser473), Alexa Fluor 555, and an Akt,PECy5 conjugated antibody to measure total levels of Akt. This two color kit is designed to measure the extent of Akt phosphorylation relative to the total Akt expression in any given cell population processed as described in the user's guide. The levels of both the total and phosphorylated protein can be measured simultaneously in the same cell, resulting in a normalized measurement of PI3K activation after stimulation. PLC/ $\mathrm{PRF} / 5$ cell line was cultured in presence of the indicated concentrations of Regorafenib, VK1, GSK1838705A and OSI-906 alone or in combination for $24 \mathrm{~h}$. Results were representative of three independent experiments. The relative graphs were created with GraphPad Prism 5.0 software.

\section{Western blot}

MAPK and PI3K/Akt signaling markers were evaluated in PLC/PRF/5 cells treated for $48 \mathrm{~h}$ as indicated above. Western Blot analysis was performed as previously described [32]. Briefly, cells were washed twice with cold PBS and then lysed in RIPA buffer (Sigma-Aldrich, Milan; Italy). After quantization of protein concentration, equal amount of protein $(50 \mu \mathrm{g})$ were resolved on SDSPAGE and transferred to polyvinyldifluoride (PVDF) filters. The blots were blocked with $5 \%(\mathrm{w} / \mathrm{v})$ nonfat dry milk for $2 \mathrm{~h}$ at room temperature and then probed with primary antibody overnight at $4{ }^{\circ} \mathrm{C}$. The primary antibodies were directed against the following proteins: IGF1-R and phospho-IGF1-R (P-IGF1-R tyr1316), p38 and phospho-p38 (P-p38 thr180/tyr182), JNK and phospho-JNK (P-JNK thr183/tyr185), TSC2 and phosphoTSC2 (Ser939), S6 and phospho-S6 (Ser235/236), AFP and $\beta$-actin (Cell Signaling, Beverly, MA, USA). After three washes, incubation was followed by the reaction with horseradish peroxidase-conjugated secondary antibody for $1 \mathrm{~h}$ at room temperature. The immunoreactive bands were visualized and analyzed using enhanced chemiluminescence detection reagents, according to the manufacturer's instructions, and chemiluminescence detection system (ChemiDoc XRS apparatus and software, Bio-Rad, Milan, Italy).

\section{Statistical analysis}

GraphPad Prism 5.0 software (La Jolla, CA, USA) was used for all statistical analysis. The significance of difference between the groups was assessed by ANOVA, followed by Kruskal-Wallis nonparametric test. $P<0.05$ was considered statistically significant. All experiments were performed in triplicate and were replicated three times. Data are presented as mean \pm standard deviation (SD).

\section{Author contributions}

BIC, CM, AC and RD - Study conception MGR - Study design

MGR,RD,CL and NC - Data acquisition

$\mathrm{CL}, \mathrm{RD}, \mathrm{AC}$ and $\mathrm{CM}$ - Data analysis and interpretation

MGR, RD, BIC, CM, NC, CL and AC - Manuscript editing.

\section{ACKNOWLEDGMENTS}

The authors acknowledge Palma Aurelia Iacovazzi of our Institute for AFP measurement and Antonio Di Carlo for generous technical support.

\section{CONFLICTS OF INTEREST}

All authors declare that there are no conflicts of interest.

\section{FUNDING}

This research was supported by Italian Ministry of Public Health (n.2/2017).

\section{REFERENCES}

1. Jemal A, Bray F, Center MM, Ferlay J, Ward E, Forman D. Global cancer statistics. CA Cancer J Clin. 2011; 61:69-90. https://doi.org/10.3322/caac.20107.

2. Llovet JM, Ricci S, Mazzaferro V, Hilgard P, Gane E, Blanc JF, de Oliveira AC, Santoro A, Raoul JL, Forner A, Schwartz M, Porta C, Zeuzem S, et al, and SHARP Investigators Study Group. Sorafenib in advanced hepatocellular carcinoma. N Engl J Med. 2008; 359:378390. https://doi.org/10.1056/NEJMoa0708857.

3. Bruix J, Qin S, Merle P, Granito A, Huang YH, Bodoky G, Pracht M, Yokosuka O, Rosmorduc O, Breder V, Gerolami R, Masi G, Ross PJ, et al, and RESORCE Investigators. Regorafenib for patients with hepatocellular carcinoma who progressed on sorafenib treatment (RESORCE): a randomised, double-blind, placebo-controlled, phase 3 trial. Lancet. 2017; 389:56-66. https://doi.org/10.1016/ S0140-6736(16)32453-9.

4. Wilhelm SM, Carter C, Tang L, Wilkie D, McNabola A, Rong H, Chen C, Zhang X, Vincent P, McHugh M, Cao Y, Shujath J, Gawlak S, et al. BAY 43-9006 exhibits broad spectrum oral antitumor activity and targets the RAF/MEK/ ERK pathway and receptor tyrosine kinases involved in tumor progression and angiogenesis. Cancer Res. 2004; 64:7099-7109. 
5. Wilhelm SM, Dumas J, Adnane L, Lynch M, Carter CA, Schütz G, Thierauch KH, Zopf D. Regorafenib (BAY 73-4506): a new oral multikinase inhibitor of angiogenic, stromal and oncogenic receptor tyrosine kinases with potent preclinical antitumor activity. Int J Cancer. 2011; 129:245-255.

6. Abou-Elkacem L, Arns S, Brix G, Gremse F, Zopf D, Kiessling F, Lederle W. Regorafenib inhibits growth, angiogenesis, and metastasis in a highly aggressive, orthotopic colon cancer model. Mol Cancer Ther. 2013; 12:1322-31. https://doi.org/10.1158/1535-7163. MCT-12-1162.

7. Dai Y, Grant S. Targeting multiple arms of the apoptotic regulatory machinery. Cancer Res. 2007; 67:2908-11.

8. Ha TY, Hwang S, Hong HN, Choi YI, Yoon SY, Won YJ, Song GW, Kim N, Tak E, Ryoo BY. Synergistic effect of sorafenib and vitamin $\mathrm{K}$ on suppression of hepatocellular carcinoma cell migration and metastasis. Anticancer Res. 2015; 35:1985-1995.

9. Carr BI, Wang Z, Wang M, Cavallini A, D'Alessandro R, Refolo MG. c-Met-Akt pathway-mediated enhancement of inhibitory c-Raf phosphorylation is involved in vitamin $\mathrm{K} 1$ and sorafenib synergy on HCC growth inhibition. Cancer Biol Ther. 2011; 12:531-538. https://doi.org/10.4161/ cbt.12.6.16053.

10. Krueger T, Westenfeld R, Schurgers L, Brandenburg V. Coagulation meets calcification: the vitamin $\mathrm{K}$ system. Int J Artif Organs. 2009; 32: 67-74.

11. Wei G, Wang M, Carr BI. Sorafenib combined vitamin k induces apoptosis in human pancreatic cancer cell lines through RAF/MEK/ERK and c-Jun NH2-terminal kinase pathways. J Cell Physiol. 2010; 224:112-119. https://doi. org/10.1002/jcp.22099.

12. Shibayama-Imazu T, Sakairi S, Watanabe A, Aiuchi T, Nakajo S, Nakaya K. Vitamin K(2) selectively induced apoptosis in ovarian TYK-nu and pancreatic MIA PaCa-2 cells out of eight solid tumor cell lines through a mechanism different from geranylgeraniol. J Cancer Res Clin Oncol. 2003; 129:1-11.

13. Yokoyama T, Miyazawa K, Naito M, Toyotake J, Tauchi T, Itoh M, You A, Hayashi Y, Georgescu MM, Kondo Y, Kondo S, Ohyashiki K. Vitamin K2 induces autophagy and apoptosis simultaneously in leukemia cells. Autophagy. 2008; 4:629-640.

14. Alexia C, Fallot G, Lasfer M, Schweizer-Groyer G, Groyer A. An evaluation of the role of insulinlike growth factors (IGF) and of type-I IGF receptor signalling in hepatocarcinogenesis and in the resistance of hepatocarcinoma cells against drug-induced apoptosis. Biochem Pharmacol. 2004; 68: 1003-1015.

15. Lippolis C, Refolo MG, D'Alessandro R, Carella N, Messa C, Cavallini A, Carr BI. Resistance to multikinase inhibitor actions mediated by insulin like growth factor-1. J Exp Clin Cancer Res. 2015; 34:90. https://doi.org/10.1186/ s13046-015-0210-1.
16. Takigawa H, Kitadai Y, Shinagawa K, Yuge R, Higashi Y, Tanaka S, Yasui W, Chayama K. Multikinase inhibitor regorafenib inhibits the growth and metastasis of colon cancer with abundant stroma. Cancer Sci. 2016; 107:601608. https://doi.org/10.1111/cas.12907.

17. Waddell T, Cunningham D. Evaluation of regorafenib in colorectal cancer and GIST. Lancet. 2013; 381: 273-275.

18. Wilhelm S, Carter C, Lynch M, Lowinger T, Dumas J, Smith RA, Schwartz B, Simantov R, Kelley S. Discovery and development of sorafenib: a multikinase inhibitor for treating cancer. Nat Rev Drug Discov. 2006; 5:835-844.

19. Berretta M, Rinaldi L, Di Benedetto F, Lleshi A, De Re V, Facchini G, De Paoli P, Di Francia R. Angiogenesis inhibitors for the treatment of hepatocellular carcinoma. Front Pharmacol. 2016; 7: 428.

20. Refolo MG, D’Alessandro R, Lippolis C, Carella N, Messa C, Cavallini A, Carr BI. Modulation of doxorubicin actions in hepatocellular carcinoma cells by Insulin-like Growth factor-I. Biochem Anal Biochem. 2016; 5:256.

21. Tovar V, Cornella H, Moeini A, Vidal S, Hoshida Y, Sia D, Peix J, Cabellos L, Alsinet C, Torrecilla S, MartinezQuetglas I, Lozano JJ, Desbois-Mouthon C, et al. Tumour initiating cells and IGF/FGF signalling contribute to sorafenib resistance in hepatocellular carcinoma. Gut. 2017; 66:530-540. https://doi.org/10.1136/gutjnl-2015-309501.

22. Chen HX, Sharon E. IGF-1R as an anti-cancer target--trials and tribulations. Chin J Cancer. 2013; 32:242-52. https:// doi.org/10.5732/cjc.012.10263.

23. Aishima S, Basaki Y, Oda Y, Kuroda Y, Nishihara Y, Taguchi K, Taketomi A, Maehara Y, Hosoi F, Maruyama Y, Fotovati A, Oie S, Ono M, et al. High expression of insulin-like growth factor binding protein-3 is correlated with lower portal invasion and better prognosis in human hepatocellular carcinoma. Cancer Sci. 2006; 97: 1182-1190.

24. D'Alessandro R, Messa C, Refolo MG, Carr BI. Modulation of sensitivity and resistance to multikinase inhibitors by microenvironmental platelet factors in HCC. Expert Opin Pharmacother. 2015; 16:2773-80. https://doi.org/10.1517/1 4656566.2015.1101065.

25. D'Alessandro R, Refolo MG, Lippolis C, Messa C, Cavallini A, Rossi R, Resta L, Di Carlo A, Carr BI. Reversibility of regorafenib effects in hepatocellular carcinoma cells. Cancer Chemother Pharmacol. 2013; 72:869-77.

26. Johnson GL, Lapadat R. Mitogen-activated protein kinase pathways mediated by ERK, JNK, and p38 protein kinases. Science. 2002; 298:1911-12.

27. Scharf JG, Schmidt-Sandte W, Pahernik SA, Ramadori G, Braulke T, Hartmann H. Characterization of the insulin-like growth factor axis in a human hepatoma cell line (PLC/ PRF/5). Carcinogenesis. 1998; 19:2121-28.

28. Chou TC. The median-effect principle and the combination index for quantitation of synergism and antagonism. In: Chou TC, Rideout DC, editors. Synergism and antagonism 
in chemotherapy. San Diego: Academic Press. 1991. pp. 61-102.

29. Chou TC, Talalay P. Quantitative analysis of dose-effect relationships: the combined effects of multiple drugs or enzyme inhibitors. Adv Enzyme Regul. 1984; 22: 27-55.

30. Berenbaum MC. Synergy, additivism and antagonism in immunosuppression. A critical review. Clin Exp Immunol. 1977; 28:1-18.

31. D'Alessandro R, Klajn A, Stucchi L, Podini P, Malosio ML, Meldolesi J. Expression of the neurosecretory process in PC12 cells is governed by REST. J Neurochem. 2008; 105:1369-1383.
32. Carr BI, D'Alessandro R, Refolo MG, Iacovazzi PA, Lippolis C, Messa C, Cavallini A, Correale M, Di Carlo A. Effects of low concentrations of Regorafenib and Sorafenib on human HCC cell AFP, migration, invasion, and growth in vitro. J Cell Physiol. 2013; 228:1344-1350.

33. Wei G, Wang M, Hyslop T, Wang Z, Carr BI. Vitamin K enhancement of sorafenib-mediated HCC cell growth inhibition in vitro and in vivo. Int $\mathrm{J}$ Cancer. 2010; 127:2949-2958.

34. Carr BI, Wang Z, Wang M, Wei G. Differential effects of vitamin $\mathrm{K} 1$ on $\mathrm{AFP}$ and DCP levels in patients with unresectable HCC and in HCC cell lines. Dig Dis Sci. 2011; 56:1876-83. 